\title{
Demographic and Clinical Characteristics of Adult Acute Myeloid Leukemia - Tertiary Care Experience
}

\author{
Sadia Sultan ${ }^{1 *}$, Hasan Abbas Zaheer ${ }^{2}$, Syed Mohammed Irfan ${ }^{1}$, Sana Ashar ${ }^{1}$
}

\begin{abstract}
Background: Acute myeloid leukemia (AML) is an acquired clonal frequent malignant disorder of myeloid progenitor cells. Our aim was to study demographical and clinicopathological features of adult Pakistani AML patients at presentation. Materials and Methods: In this single centre study extending from January 2010 to December 2014, data were retrieved from the patient records with a predetermined performa and analyzed with SPSS version 22. Results: Overall 125 patients were diagnosed at our institution with de novo AML during the study period. There were 76 males and 49 females (ratio 1.5:1), with an age range between 15 and 85 years and a mean age of $38.8 \pm 20.1$ years. The major complaints were fever $(72.8 \%)$, generalized weakness $(60 \%)$, bleeding $(37.6 \%)$ and dyspnea $(12 \%)$. Physical examination revealed pallor in $\mathbf{5 6 . 8 \%}$, splenomegaly and hepatomegaly in $16 \%$ and $12.8 \%$, respectively, and lymphodenopathy in $10.4 \%$. The mean hemoglobin was $8.19 \pm 2.12 \mathrm{~g} / \mathrm{dl}$ with a mean MCV of $86.0 \pm 9.83 \mathrm{fl}$, a mean total leukocyte count of $43.1 \pm 68.5 \times 10^{9} / 1$, an $\mathrm{ANC}$ of $3.09 \pm 6.66 \times 10^{9} / \mathrm{l}$ and a mean platelet count of $62.3 \pm 78.6 \times 10^{9} / 1$. Conclusions: AML in Pakistani patients is seen in a relatively very young population with male preponderance, compared with the west. However, clinico-pathological features appear comparable to published data.
\end{abstract}

Keywords: Acute myeloid leukemia - demographics - clinical characteristics - Pakistan.

Asian Pac J Cancer Prev, 17 (1), 357-360

\section{Introduction}

Leukemias are heterogeneous group of acquired clonal hematological malignancies that incorporates diverse biologically distinct subgroups. Adult acute myeloid leukemia (AML) represents one of the most biologically and clinically diverse phenotypic disease amongst various hematopoietic malignancies. (Ahmad et al., 2014).

AML is the most frequent adult's acute leukemia (Zhou et al., 2013). An estimated that annually, more than one quarter of a million adults throughout the world are diagnosed with acute myeloid leukemia (Rowe and Tallman, 2010). In the available literature annual incidence for AML is 3-4/100,000 population per year (Schlenk., 2014). The incidence of AML increases with age and median age of patients with acute myeloid leukemia is around 70 years (Ossenkoppele and Lowenberg, 2015). Statistics show that more than half of patients with AML are older than 60 years (Deschler and Lubbert, 2006).

AML is genetically typified by the accretion of somatically acquired genetic changes in hematopoietic progenitor cells altering normal mechanisms of proliferation and differentiation. (Schlenk., 2014). Thus resulting in accumulation of myeloid lineage blasts cells in the bone marrow (Estey, 2014). These cells interfere with normal hematopoiesis, contributing to the bone marrow failure, which is the most common underlying cause of mortality. Several risk factors and chromosomal aberration have been identified, but the specific underlying cause is not clear.

The clinical biology of AML among patients is highly heterogeneous (Yang et al., 2012; Su et al., 2014). The symptoms of AML are predominantly caused by replacement of normal bone marrow with leukemic cells, which causes a rapid decline in normal peripheral blood counts. These symptoms often include fever, fatigue, dyspnea, easy bruising, bleeding and frequent infections. Visceromegaly may occur in AML, but it is typically mild and mostly asymptomatic.

To date very little information is available from our country on this disorder. Research on epidemiological features of AML is poorly reported from Pakistan. The purpose of this study was to demonstrate demographic and clinico-pathological features of AML patients who visited our tertiary care center from 2010 to 2014.

\section{Materials and Methods}

This is descriptive cross sectional study done at hematology department of Liaquat National Hospital. The study was conducted over a period of 5 years from January 2010 to December 2014.

Patients having history of preceding hematological disorders like Myelodysplastic syndrome, Chronic 
myeloid leukemia, Myeloproliferative neoplasm and with prior history of chemotherapy/radiotherapy were excluded from the study. The patients who were known cases of relapse/refractory AML were also not included. Based on this, a total of 125 subjects with newly diagnosed untreated de novo AML were included in the study. The diagnosis of AML was ascertained according to the standard FAB criteria, and was based on bone marrow morphology and cytochemistry (Bennett et al., 1985).

Complete blood counts were done on Cell Dyne counter (Abbott, USA). Bone marrow aspiration was done from posterior iliac crest through Jamshidi needle and was stained by Leishman's stain. Cytochemical stains were carried out on each bone marrow smears including Sudan Black B (SBB), Periodic acid-Schiff (PAS) and Alpha naphthyl acetate esterase by commercially provided kits from Merck Diagnostic according to manufacturer's instructions. Immunophenotyping was done where it was deemed necessary, in patients with diagnostic uncertainty.

Approval from the institutional ethical and research review committee was obtained prior to the study.

\section{Data analysis}

Data was compiled and analyzed using the Statistical Package for the Social Sciences version 22.0 (SPSS Inc, Chicago, IL, USA). The results were expressed as mean $\pm \mathrm{SD}$ for quantitative variables and qualitative variables are presented as frequency \& percentages.

\section{Results}

Out of 125 patients, 76 were males $(60.8 \%)$ and 49 were females (39.2\%) with male to female ratio of 1.5:1. Age ranged between 15 and 85 years with a mean age of $37.97 \pm 20.12$ years and median age of 34.5 years. Overall 95 (76\%) patients were under 50 years of age, and remaining only $24 \%$ (30 patients) were above 50 years. Age stratification is shown in Table-1.

The major complaints were fever in $91(72.8 \%)$ patients; generalized weakness in $75(60 \%)$ patients; bleeding in $47(37.6 \%)$ patients and dyspnea in $15(12 \%)$ patients. The most frequent sites of bleeding were skin, gums and nose. None of the patients had history of intracranial bleeding.

Physical examination revealed pallor as a predominant finding detected in $71(56.8 \%)$ patients followed splenomegaly and hepatomegaly in $20(16 \%)$ and 16 (12.8\%) patients respectively. Lymphodenopathy was noted in $13(10.4 \%)$ patients.

The mean hemoglobin was 8.19 \pm 2.12 (range 3.7-13.6)

Table 1.Age Stratification in Adult Patients with Acute Myeloid Leukemia

\begin{tabular}{lcccc}
\hline Age groups & $\begin{array}{c}\text { Male } \\
\mathrm{n}=76\end{array}$ & $\begin{array}{c}\text { Female } \\
\mathrm{n}=49\end{array}$ & $\begin{array}{c}\text { Total } \\
\mathrm{n}=125\end{array}$ & $\begin{array}{c}\text { Percentage } \\
\%\end{array}$ \\
\hline $15-30$ & 37 & 20 & 57 & 45.4 \\
$31-50$ & 20 & 18 & 38 & 30.4 \\
$51-65$ & 10 & 7 & 17 & 13.6 \\
$66-85$ & 9 & 4 & 13 & 10.4 \\
\hline
\end{tabular}

$\mathrm{g} / \mathrm{dl}$ with the mean MCV of $85.98 \pm 9.83 \mathrm{fl}$. The mean total leukocyte count of $43.08 \pm 68.45$ (range 0.6-372) $\mathrm{x} 10^{9} / 1$; Absolute neutrophilic count (ANC) of 3.09 \pm 6.66 (range 0.01-15.4) $\times 10^{9} / 1$ and mean platelets count were 62.32 \pm 78.61 (range 3.0-576) x10\%/1.

Anemia $(\mathrm{Hb}<10 \mathrm{gm} / \mathrm{dl})$ was noted in $102(81.6 \%)$ patients. Hyperleukocytosis (TLC count $>100 \times 10^{9} / 1$ ) was seen in $19(15.2 \%)$ patients. Thrombocytopenia (platelets count $\left.<100 \times 10^{9} / 1\right)$ was detected in $105(84 \%)$ patients, while severe thrombocytopenia (platelets $<20 \times 10^{9} / 1$ ) was seen in $33(26.4 \%)$ patients.

\section{Discussion}

Acute myeloid leukemia is an aggressive, clonal neoplasm, with maturation arrest in granulopoiesis, resulting in an accretion of immature myeloblasts in the bone marrow. It progresses rapidly and is fatal within weeks or months if left untreated. The present study has illustrated demographics, clinical features and hematological markers in Pakistani AML patients.

AML can occur in patients of any age, but in general the overall incidence increases with age. AML is a disease of the elder, with a median age of onset around $\sim 70$ years (Juliusson et al., 2009). According to the National comprehensive cancer network (NCCN), 54\% of patients diagnosed at 65 years or older and approximately a third diagnosed at $\geq 75$ years of age (Yin et al., 2012). Surprisingly, the median age of the patients in our study is 34.5 years. Previously published studies, about a decade back, from Pakistan also revealed comparable age at presentation; 32 and 38 years respectively (Kakepoto et al., 2002; Harani et al., 2005).

Similar to us a large regional study reported by Chauhan et al from neighbor India, revealed the mean age of AML patients as 32 years at disease presentation (Chauhan et al., 2013). One recent Malaysian study by Meng et al reported the median age of 39 years at diagnosis (Meng et al., 2013). When compared with earlier international reports, our results are in conflict with studies published from Sweden and Germany, where the median age were 71 and 60 years respectively. (Lazarevic, 2014; Pastore, 2014). Perhaps this disparity may be clarified by obvious difference based on geographical and genetic makeup between two populations and also accountable is the higher mean age in western countries compared to us.

AML is slightly more common in men, with a male to female ratio of $\sim 2: 1$. In the present study male preponderance was observed and it was consistent with other studies (Harani et al., 2005; Chauhan et al., 2011).

In general, diminution in hematopoiesis owing to marrow proliferation of myeloblast cells, results in anemia, thrombocytopenia and leucopenia with the clinical symptoms of pallor, easy fatigue, bleeding manifestations and fever respectively.

Among our patients majority presented with fever and generalized weakness. Similar presenting symptoms were observed by prior Indian and Pakistani studies conducted by Preethi and Kakepoto et al (Kakepoto et al., 2002; CRP, 2014). Clinical features in our study somewhat correlated with the study conducted by Khalid et al. (1997) showing 
$59 \%$ of patients had febrile illness compared with us having prevalence of $72.8 \%$ (Khalid et al., 1997). Bleeding was also a common presenting feature in our patients. Comparable findings have been reported in other studies on AML. When compared with earlier reports, our results are in concurrence with a study which reported fever and bleeding diathesis in $80.4 \%$ and $44.5 \%$ respectively (Asif and Hassan, 2013).

Pallor was found as a presenting symptom in $56.8 \%$ of the patients. This correlates with the study conducted by Harakati et al from Saudi Arabia revealed pallor in 69\% of their patient's series (Harakati et al., 1998).

In Acute myeloid leukemia extramedullary infiltrates by leukemic cells may causes visceromegaly or lymphadenopathy. Though lymphadenopathy is not as common as seen in acute lymphoblastic leukemia. Rather hepatosplenomegaly is more frequent but massive visceromegaly is very rare. In our patients, hepatomegaly and splenomegaly were observed in $12.8 \%$ and $16 \%$ patients respectively. Majority had mild to moderate visceromegaly. Lymph node enlargement was noted in $10.4 \%$ of our patients. Our results are comparable with other study reported from Yemen which revealed $20 \%$ of patients had lymphodenopathy (Hamid and Nabhi, 2015).

Anemia is a constant feature in all acute leukemias and in majority of cases is due to bone marrow infiltration. Evaluations of hematological parameters in our cases predominantly showed low mean hemoglobin levels. Our results are analogous with other studies (Ghosh et al., 2003; Naseem et al., 2013).

Among all adult patients with acute leukemias, approximate 5 to $30 \%$ may present with symptoms of leukostasis and hyperleukocytosis. Hyperleukocytosis are much more pronounced in AML than in ALL (Asif and Hassan., 2013). In our study $15.2 \%$ of patients showed hyperleukocytosis. Analogous to this finding one regional study from Islamabad revealed $11 \%$ prevalence of hyperleukocytosis in AML patients (Asif and Hassan., 2013).

Lastly thrombocytopenia is an important well known manifestation of acute leukemias. Thrombocytopenia was present in the majority $(84 \%)$ of cases in the present study. In concurrence to our results regional study (86\%) revealed more or less similar findings (Naseem et al., 2013).

So in conclusion, AML is predominantly seen in very younger age group in Pakistan. At disease presentation age is approximately half that is reported in international data. However clinico-epidemiological features are appearing comparable to published data.

\section{References}

Ahmad F, Mohota R, Sanap S, et al (2014). Molecular evaluation of DNMT3A and IDH1/2 gene mutation: frequency, distribution pattern and associations with additional molecular markers in normal karyotype Indian acute myeloid leukemia patients. Asian Pac J Cancer Prev, 15, 1247-53.

Asif N, Hassan K (2013). Acute myeloid leukemia amongst adults. J Islamabad Med Dental College, 2, 58-63.

Bennett JM, Catovsky D, Daniel MT, et al (1985). Proposed revised criteria for the classification of acute myeloid leukemia. A report of the French-American-British Cooperative Group. Ann Intern Med, 103, 620-5.

Chauhan PS, Ihsan R, Singh LC, et al (2013). Mutation of NPM1 and FLT3 genes in acute myeloid leukemia and their association with clinical and immunophenotypic features. Dis Markers, 35, 581-8.

Chauhan PS, Bhushan B, Mishra AK, et al (2011). Mutation of FLT3 gene in acute myeloid leukemia with normal cytogenetics and its association with clinical and immunophenotypic features. Med Oncol, 28, 544-51.

C R P (2014). Clinico-hematological study of acute myeloid leukemias. J Clin Diagn Res, 8, 14-7.

Deschler B, Lubbert M (2006). Acute myeloid leukemia: epidemiology and etiology. Cancer, 107, 2099-107.

Estey EH (2014). Acute myeloid leukemia: 2014 update on riskstratification and management. Am J Hematol, 89, 1063-81.

Ghosh S, Shinde SC, Kumaran GS, et al (2003). Hematologic and immunophenotypic profile of acute myeloid leukemia: an experience of tata memorial hospital. Indian J Cancer, 40, 71-6.

Harani MS, Adil SN, Shaikh MU, et al (2005). Frequency of fab subtypes in acute myeloid leukemia patients at Aga Khan University Hospital Karachi. J Ayub Med Coll Abbottabad, 17, 26-9.

Hamid GA, Nabhi A (2015). Clinicoepidemiological features of adult leukemias in Aden, Yemen. Indian J Applied Res, 5, 334-6.

Harakati MS, Al-Momen AM, Ajarim DS, et al (1998). Adult acute myeloblastic leukemia: experience at king khalid university hospital. Ann Saudi Med, 18, 221-5.

Juliusson G, Antunovic P, Derolf A, et al (2009). Age and acute myeloid leukemia: real world data on decision to treat and outcomes from the Swedish Acute Leukemia Registry. Blood, 113, 4179-87.

Kakepoto GN, Burney IA, Zaki S, et al (2002). Long-term outcomes of acute myeloid leukemia in adults in Pakistan. J Pak Med Assoc, 52, 482-6.

KhalidA,Zahid M,RehmanA,etal (1997).Clinicoepidemiological features of adult leukemias in Pakistan. J Pak Med Assoc, 47, 119-22.

Lazarevic V, Horstedt AS, Johansson B, et al (2014). Incidence and prognostic significance of karyotypic subgroups in older patients with acute myeloid leukemia: the Swedish population-based experience. Blood Cancer J, 28, 4-188.

Meng CY, Noor PJ, Ismail A, et al (2013). Cytogenetic profile of de novo acute myeloid leukemia Patients in Malaysia. Int J Biomed Sci, 9, 26-32.

Naseem N, Imtiaz U, Mobeen S (2013). Evaluation of frequency and clinico-hematological features of acute myeloid leukemia at a tertiary care hospital, lahore. Pakistan J Med Health Sci, 7, 347-9.

Ossenkoppele G, Lowenberg B (2015). How I treat the older patient with acute myeloid leukemia. Blood, 125, 767-74.

Pastore F, Dufour A, Benthaus T, et al (2014). Combined molecular and clinical prognostic index for relapse and survival in cytogenetically normal acute myeloid leukemia. J Clin Oncol, 20, 1586-94.

Schlenk RF (2014). Post-remission therapy for acute myeloid leukemia. Haematologica, 99, 1663-70.

Su L, Li X, Gao SJ, et al (2014). Cytogenetic and genetic mutation features of de novo acute myeloid leukemia in elderly Chinese patients. Asian Pac J Cancer Prev, 15, 895-8.

Rowe JM, Tallman MS (2010). How I treat acute myeloid leukemia. Blood, 116, 3147-56.

Yang XF, Sun AN, Yin J, et al (2012). Monosomal karyotypes 
Sadia Sultan et al

among 1147 Chinese patients with acute myeloid leukemia: prevalence, features and prognostic impact. Asian Pac J Cancer Prev, 13, 5421-6.

Yin JA, O'Brien MA, Hills RK, et al (2012). Minimal residual disease monitoring by quantitative RT-PCR in core binding factor AML allows risk stratification and predicts relapse: results of the United Kingdom MRC AML-15 trial. Blood, 120, 2826-35.

Zhou L, Zhu YY, Zhang XD, et al (2013). Risk effects of GST gene polymorphisms in patients with acute myeloid leukemia: a prospective study. Asian Pac J Cancer Prev, 14, 3861-4. 